\title{
Automated Training Data Generation for Microscopy Focus Classification
}

\author{
Dashan Gao ${ }^{1}$, Dirk Padfield ${ }^{1}$, Jens Rittscher ${ }^{1}$, and Richard McKay ${ }^{2}$ \\ 1 GE Global Research, One Research Circle, Niskayuna, NY, 12309 \\ \{gaoda,padfield, rittsche\}@ge.com \\ 2 Omnyx, 800 Centennial Avenue, Building 4, 2nd Floor, Piscataway, NJ 08855 \\ Richard.McKay@omnyx.com
}

\begin{abstract}
Image focus quality is of utmost importance in digital microscopes because the pathologist cannot accurately characterize the tissue state without focused images. We propose to train a classifier to measure the focus quality of microscopy scans based on an extensive set of image features. However, classifiers rely heavily on the quality and quantity of the training data, and collecting annotated data is tedious and expensive. We therefore propose a new method to automatically generate large amounts of training data using image stacks. Our experiments demonstrate that a classifier trained with the image stacks performs comparably with one trained with manually annotated data. The classifier is able to accurately detect out-of-focus regions, provide focus quality feedback to the user, and identify potential problems of the microscopy design.
\end{abstract}

\section{Introduction}

The adoption of digital microscopes in pathology studies enables the digitization of large tissue slides, which can then be stored, transferred, and analyzed electronically. As a crucial component of a digital microscope, the quality of the autofocus system directly determines the quality of the tissue scans. If the image is unfocused, the user will not be able to accurately characterize the tissue state as either diseased or healthy, and poor focus quality dampens the user's experience and can cause the user to loose confidence in the system. There is therefore a demand for developing focus quality assessment tools that detect such focus errors in whole slide images (WSI). Such ability to automatically measure image focus quality provides feedback to the pathologist as to the quality of the acquisition, and when such measures are fed back to the microscope itself, the system is able to correct problems even before the pathologist sees it.

Many approaches have been developed previously for predicting the focus plane for tissue imaging. Most of these approaches calculate some features on the image taken at a given microscope depth. For example, Liron et al. [1] measured the intensity of the reflected red laser light, Santos et al. 2] used various image gradient and histogram based metrics, Firestone et al. 3] compared measurements of the entropy and the range of the image intensity, and the authors

T. Jiang et al. (Eds.): MICCAI 2010, Part II, LNCS 6362, pp. 446-45, 2010.

(C) Springer-Verlag Berlin Heidelberg 2010 
of [4/5] adopted the normalized variance of image intensity. Other features include the sum of the absolute value of the image Laplacian [6] and the integrated amplitude modulus [7. After the features are measured at one depth, the microscope depth is shifted, and the features are calculated again. If calculations are made at a sufficient number of depth locations and these locations span the actual location of the optimal focus, they will be sufficient to calculate the correct focus plane 899 . The requirement to acquire multiple images through the true focal plane can be prohibitively time consuming, especially for a highthroughput system. Furthermore, when the image quality measure is made on images after the acquisition step, the intermediate images may not be available, so the quality must be assessed on a single image.

To enable focus quality measurement on a single image, we trained classifiers to divide the image into in-focus and out-of-focus regions. This framework is considered a "no reference" approach since it enables the measurement of focus quality without the need for other images. Classifiers rely heavily on the quality and quantity of the training data, which is expensive and tedious for pathologists to generate, so we introduce a method of automatically generating training data using image stacks. Our quantitative experiments on annotated dataset and image stacks demonstrate that the classifiers can robustly separate in-focus from out-of-focus regions, provide useful feedback to the user about the focus quality, and even identify issues in the microscopy design such as stage tilt.

\section{Region Classification for Focus Quality Assessment}

We treat the image focus quality assessment as a binary classification problem, wherein the goal is to classify each WSI as either in-focus or out-of-focus. Guidance from pathologists indicate that a useful measure of focus quality is to divide the WSI into regions, classify each region as in-focus or out-of-focus, and calculate the percentage of in-focus regions. Some advantages of classifying smaller regions are that the image slide may be slightly tilted so that the focus depth varies slowly across the image, and it is likely that only part of the entire image is out-of-focus since the microscope does not fail completely for a scan in many cases. Therefore, we evenly divide each WSI into smaller regions and classify each region independently.

For classification, we represent an image region $I$ by a vector of features, the choice of which is an important step to avoid features that bias away from the true focus [10. We therefore utilize an extensive set of 44 features, most of which have been studied or suggested in previous microscope auto-focusing works [10]3[11]. The definitions and brief descriptions of these features are given in Table1. We then adopt an AdaBoost classifier, which combines the outputs of a set of sequentially selected weak classifiers into a strong classifier to minimize the overall classification risk [1213. In our implementation, the weak classifier is generated by the linear discriminant analysis [14].

In general, the samples for training the classifier are manually cropped from images through careful visual inspection by expert pathologists. However, we 
Table 1. Feature equations and descriptions. $x$ and $y$ are image coordinates. $M$ and $N$ are the width and the height of the image, respectively.

\begin{tabular}{|c|c|}
\hline \multicolumn{2}{|c|}{$\begin{array}{c}\text { Neighborhood contrast features (24) } \\
\text { Mean, variance, skew, and kurtosis of contrast calculated for each neighborhood } \\
\text { Neighborhood } \mathcal{N} \text { sizes: } 5 \times 5,9 \times 9, \text { and } 17 \times 17\end{array}$} \\
\hline $\begin{array}{l}\text { Neighborhood }(\mathcal{N}) \text { contrast } \\
\text { Normalized neighborhood }(\mathcal{N}) \text { contrast }\end{array}$ & $\begin{array}{l}\max _{(x, y) \in \mathcal{N} I(x, y)-\min _{(x, y) \in \mathcal{N}} I(x, y)} \\
\frac{\max _{(x, y) \in \mathcal{N} I(x, y)-\min _{(x, y) \in \mathcal{N}} I(x, y)}}{\max _{(x, y) \in \mathcal{N}} I(x, y)}\end{array}$ \\
\hline \multicolumn{2}{|c|}{$\begin{array}{l}\text { Gradient and Laplacian features (3) } \\
S_{x} \text { and } S_{y} \text { are the Sobel operators. } \\
L_{y} \text { are Laplacian filter output in } x \text { and } y \text { directions. }\end{array}$} \\
\hline 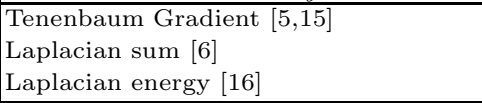 & $\begin{array}{l}\frac{1}{M N} \sum_{x} \sum_{y} S_{x}(x, y)^{2}+S_{y}(x, y)^{2} \\
\frac{1}{M N} \sum_{x} \sum_{y}\left|L_{x}(x, y)\right|+\left|L_{y}(x, y)\right| \\
\frac{1}{M N} \sum_{x} \sum_{y}\left(L_{x}(x, y)+L_{y}(x, y)\right)^{2}\end{array}$ \\
\hline \multicolumn{2}{|c|}{$\begin{array}{l}\text { Local image statistics features (7) } \\
\quad \mu=\frac{1}{M N} \sum_{x} \sum_{y} I(x, y) \text { is the average of image intensity. } \\
p_{i} \text { is the probability of observing a pixel of intensity } i \text { in the image. }\end{array}$} \\
\hline 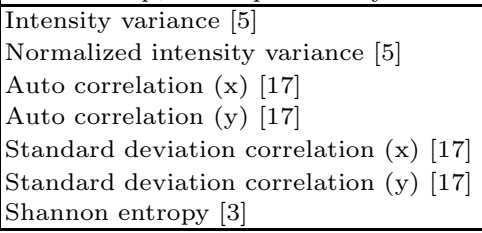 & $\begin{array}{l}\frac{1}{M N} \sum_{x} \sum_{y}(I(x, y)-\mu)^{2} \\
\frac{1}{M N \mu} \sum_{x} \sum_{y}(I(x, y)-\mu)^{2} \\
\frac{1}{M N} \sum_{x} \sum_{y} I(x, y)(I(x+1, y)-I(x+2, y)) \\
\frac{1}{M N} \sum_{x} \sum_{y} I(x, y)(I(x, y+1)-I(x, y+2)) \\
\frac{1}{M N} \sum_{x} \sum_{y} I(x, y) I(x+1, y)-\mu^{2} \\
\frac{1}{M N} \sum_{x} \sum_{y} I(x, y) I(x, y+1)-\mu^{2} \\
-\sum_{i} p_{i} \log _{2}\left(p_{i}\right) \\
\end{array}$ \\
\hline $\begin{array}{r}\text { Wav } \\
\text { Image decomposed into } \\
W_{k} \text { is a wave } \\
E_{a} \text { is the energy of } A, E_{d} \\
\alpha_{k} \text { and } \beta_{k} \text { are the par } \\
\text { distribution }(C\end{array}$ & $\begin{array}{l}\text { let features (10) } \\
\text { pproximation } A \text { and detail } D \text { images. } \\
\text { et channel with } k \in 0,1,2 \\
k \text { ) is the energy of the } k_{t h} \text { detail image } \\
\text { meters for the generalized Gaussian } \\
\text { GD) representation for } W_{k} \\
\end{array}$ \\
\hline $\begin{array}{l}\text { Channel energy } \\
\text { Defocus ratio } 11\end{array}$ & $\begin{array}{l}\frac{1}{M N} \sum_{x} \sum_{y}\left|W_{k}(x, y)\right| \\
\frac{E_{a}}{E_{d}(1)+E_{d}(2)} \\
P\left(W_{k}(x, y)\right)=\frac{\beta_{k}}{2 \alpha_{k} \Gamma\left(1 / \beta_{k}\right)} \exp \left\{-\left(\frac{\left|W_{k}(x, y)\right|}{\alpha_{k}}\right)_{k}^{\beta}\right\}\end{array}$ \\
\hline
\end{tabular}

will demonstrate that this tedious and slow process can be expedited by a clever utilization of tissue image stacks. Finally, given the in-focus/out-of-focus assignments from the classifier for all regions in a WSI, the focus quality is measured as the percentage of the regions that are out-of-focus. In addition, if there is time for re-acquisition, the images of the worst focus can be re-acquired.

\section{Automatic Training Data from Image Stacks}

To generate training data, images were manually collected from various tissue scans by two pathologists. The dataset consisted of image patches at two different magnification levels: $20 \times$ and $40 \times$. For each magnification level, they labeled approximately 30 in-focus and 30 out-of-focus regions with the average size around $200 \times 200$ pixels. We extracted the 44 features from Table 1 for every image region covered by a $128 \times 128$ window, placed at intervals of 16 pixels in both $x$ and $y$ directions. Although this dataset was annotated by experts, a drawback is that its size is limited because the process of selecting such regions 


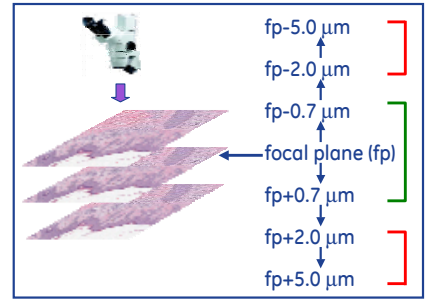

Acquisition setup

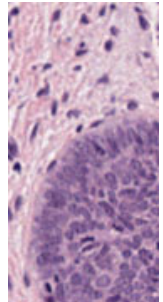

$\mathrm{fp}-2 \mu m$

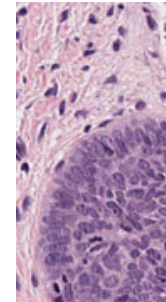

focal plane

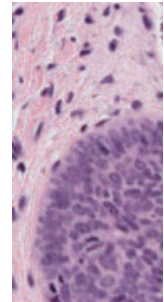

$\mathrm{fp}+2 \mu m$

Fig. 1. Illustration of the generation of training data from image stacks. Images within $0.7 \mu \mathrm{m}$ of the focal plane are considered in-focus, and images between $2.0 \mu \mathrm{m}$ and $5.0 \mu \mathrm{m}$ away from the focal plane are considered out-of-focus.

is tedious and time-consuming. Trained with a small number of samples, the generalization of the classifier is limited.

To overcome the limited amount of data, we developed a method of automatically generating large amounts of training samples using image stacks. Image stacks are a set of images of the same tissue taken at different depths, with the focal plane manually determined. Figure 1 shows the general setup. According to pathologists, they consider a small range of slices around the focal plane to be in focus, and a range of slices far from the focal plane to be out-of-focus. Based on experience, they suggested the following thresholds: between $-0.7 \mu \mathrm{m}$ and $0.7 \mu \mathrm{m}$ was selected as "in-focus", and between $2.0 \mu \mathrm{m}$ and $5.0 \mu \mathrm{m}$ and between $-5.0 \mu \mathrm{m}$ and $-2.0 \mu \mathrm{m}$ were selected as "out-of-focus". The images between $0.7 \mu \mathrm{m}$ and $2.0 \mu \mathrm{m}$ and between $-2.0 \mu \mathrm{m}$ and $-0.7 \mu \mathrm{m}$ were determined to be too subtle to be accurately measured, and the images beyond $\pm 5.0 \mu \mathrm{m}$ are too out-of-focus to be useful in practice.

Using these thresholds, we generated an expanded image set that consisted of 37 tissue stacks, each of which includes 77 images scanned from $-5 \mu m$ to $-2 \mu m,-0.7 \mu m$ to $0.7 \mu m$, and $2 \mu m$ to $5 \mu m$ with an incremental step of $0.1 \mu m$. The focus plane of each stack was carefully selected and verified by an expert before and after scanning. Each microscope image had a size of $2048 \times 2048$ pixels and was evenly divided into 256 non-overlapping samples each of size $128 \times 128$. Altogether, this led to 142,080 positive samples and 587,264 negative samples.

\section{Experiments and Results}

We have tested various parameters, such as the slide window size (128 or 256), and the initial weights for positive and negative samples, but we did not observe significant changes in the classification accuracy, which suggests the robustness of the classifier. For the results presented in the following, we chose a window size of 128 pixels, and equal initial weights for positive and negative samples. Table 2 provides a comparison of the classification accuracy of the AdaBoost classifiers trained with either the manually labeled samples or the image stacks. 
Table 2. The average training and testing accuracy for a 10 -fold cross validation on the manually labeled dataset (ManualSet) and the automatically generated dataset (StackSet). Both sets had high accuracy, but StackSet achieved better results than ManualSet, especially for $40 \times$ magnification. The training accuracy is the classification accuracy when testing on the training data (which gives an upper bound on the accuracy), and the testing accuracy represents the result on new test data. The test accuracy is close to the training accuracy, indicating no over-fitting during training.

\begin{tabular}{|c|c|c|c|c|c|c|}
\hline & \multicolumn{2}{|c|}{ ManualSet-100iter. } & \multicolumn{2}{|c|}{ ManualSet-30iter } & \multicolumn{2}{|c|}{ StackSet-30iter. } \\
\hline & $20 \mathrm{X}$ & $40 \mathrm{X}$ & $20 \mathrm{X}$ & $40 \mathrm{X}$ & $20 \mathrm{X}$ & $40 \mathrm{X}$ \\
\hline Training Accuracy (\%) & 91.939 & 87.062 & 91.918 & 86.319 & 92.806 & 93.421 \\
\hline Testing Accuracy (\%) & 90.854 & 86.679 & 90.996 & 85.683 & 92.772 & 93.371 \\
\hline
\end{tabular}

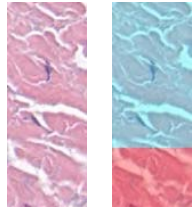

(a)

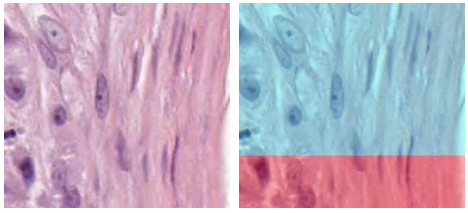

(b)
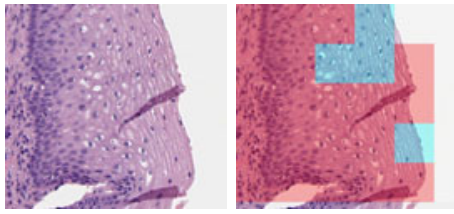

(c)

Fig. 2. Illustration of the sensitivity of the classifier trained with stacks. The classification results were overlaid on the images which were labeled by pathologists as $(\mathrm{a}, \mathrm{b})$ "in-focus" and (c) "out-of-focus". The overlaid blue and red colors represent in-focus and out-of-focus assignments, respectively. Closer inspection indicated that the focus quality indeed varies throughout the tissue, and the classifier were able to detect this.

The average training and testing accuracy are from a 10 -fold cross validation, where we randomly, but evenly, divided the samples into ten subsets, and in each round, we chose nine of them for training and one for testing. For each classifier, 30 boosting iterations were considered, and we also show 100 boosting iterations for the manual set. In addition, two different magnification levels of $20 \times$ and $40 \times$ were trained separately because the characteristics of tissue images under these magnifications are significantly different.

The table indicates the advantage of the proposed automatic data generation method. First, the classifiers obtained using the automatically generated data (StackSet-30iter) achieved better accuracy than those trained with the manual data for both $20 \times$ and $40 \times$ images. In particular, for $40 \times$ images the improvement is nearly $8 \%$, indicating the usefulness of the vast amount of training samples provided by the image stacks method. Second, while the performance gap between $20 \times$ and $40 \times$ images for the manual data indicated that the $40 \times$ class is more difficult than the $20 \times$ class, the gap was bridged by using the stacks. Third, the classification accuracy does not improve much by increasing the training iterations to 100 for the manual data (ManualSet-100iter) suggesting that the poorer accuracy results from the limited amount of annotated data. Thus, the table demonstrates that using image stacks to automatically generate training data is an effective method and leads to impressive accuracy. 


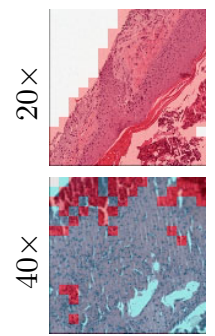

$-3 \mu m$

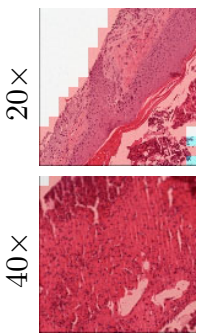

$-3 \mu m$

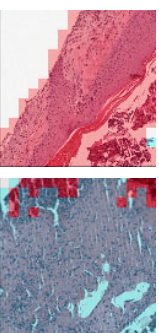

$-2 \mu m$

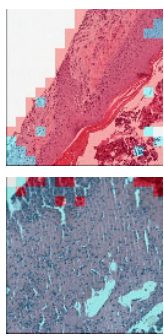

$-1 \mu m$

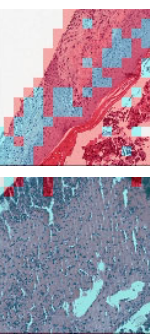

$0 \mu m$

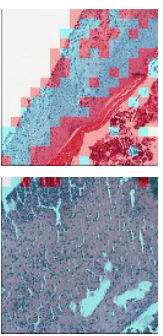

$1 \mu \mathrm{m}$

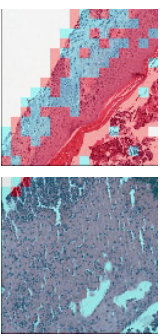

$2 \mu \mathrm{m}$

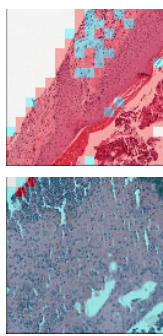

$3 \mu m$

(a) Result of training with manually annotated data.

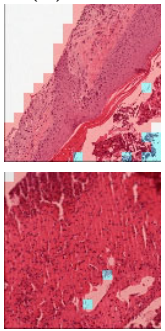

$-2 \mu m$
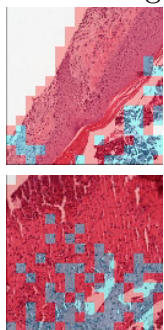

$-1 \mu \mathrm{m}$
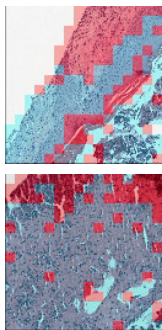

$0 \mu \mathrm{m}$
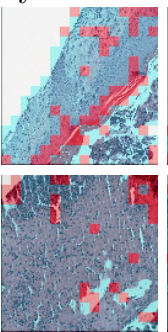

$1 \mu \mathrm{m}$

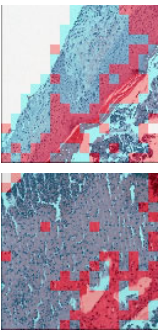

$2 \mu m$
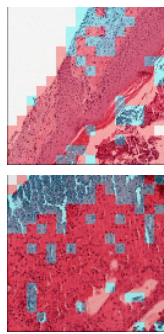

$3 \mu \mathrm{m}$

(b) Result of training with image stacks.

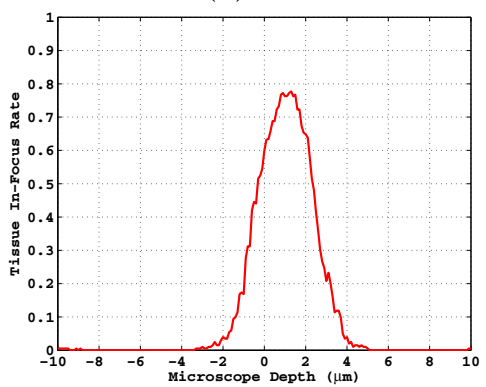

(c) $20 \times$ in-focus measure

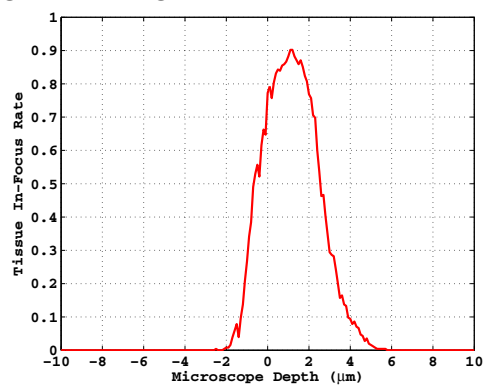

(d) $40 \times$ in-focus measure

Fig. 3. (a) Classification results on $20 \times$ and $40 \times$ stacks using classifiers trained with manually labeled data. The blue color represents in-focus assignment, and the red represents out-of-focus assignment. Ideally, a region is classified as in-focus near its focal plane, and as out-of-focus when far away. However, the lower-right regions of the $20 \times$ stack were always classified as out-of-focus, while almost all regions of the $40 \times$ stack were classified as in-focus. This indicates the poor generalization of the classifiers. (b) Classification results on the same $20 \times$ and $40 \times$ stacks as in (a) using classifiers trained with automatically generated data. The classification results show a clear trend throughout both stacks of each region going from out-of-focus to in-focus and back. (c) and $(\mathrm{d})$ show plots of the percentage of in-focus tissue regions over each entire stack. The classification was carried out independently for each image of the stack, but they still show a clear and smooth trend of increasing towards the focal plane at $1 \mu \mathrm{m}$ as expected for the image stacks. 
In Figure 2, we show some classification results overlaid on example test images, which were labeled by the pathologists as (a,b) "in-focus" and (c) "outof-focus". The classifier indicated that the images were not fully in-focus or out-of-focus but rather included a mixture of in-focus and out-of-focus regions, and a closer inspection confirmed this. For example, the top-right portion of the tissue in Figure 2(c) (shown in blue) was in clear focus. The automatic classifier was sensitive enough to detect such labeling ambiguities.

To evaluate the generalization of the trained classifiers, we tested them on a $20 \times$ and a $40 \times$ image stack that were not used previously in either training or testing. Figure 3 shows these images scanned at different microscope depths overlaid with the classification results. Ideally, the in-focus/out-of-focus assignments by a classifier would change consistently along the stack, so that a region is classified as out-of-focus when scanned far away from the focal plane, and as in-focus when scanned at or close to the focal plane. However, as illustrated in Figure 3(a), the classifiers trained with the manual set generalized poorly in most of the regions. For example, it always classified the lower-right tissue regions of the $20 \times$ stack as out-of-focus and classified almost all regions of the $40 \times$ stack as in-focus. On the other hand, as shown in Figure 3(b), the classifiers trained with image stacks produced consistent results showing that most regions in both $20 \times$ and $40 \times$ stacks went from out-of-focus to in-focus and to out-of-focus again along the depth axis. In Figure 3(b), a small portion of tissues in the $20 \times$ scans was classified at out-of-focus throughout the entire stack. The pathologists verified that these are the edge regions of the tissue which lie at a different focal plane than the tissue and are indeed out-of-focus throughout the entire stack.

Figure 3(b) also shows that, in both stacks, as the stack images go from outof-focus to in-focus, the in-focus regions propagate from the lower-right corner to the upper-left. This phenomenon seemed to indicate a problem that the stage which holds the tissue slide was not orthogonal to the optical axis of the camera. We reported this stage tilt problem that was automatically identified by the algorithms to the microscope design team, and they verified and corrected this issue. Thus, the classification results provided not only a means of feedback to the user but also crucial information for improving the microscope design.

Finally, we show the classification results for the WSI as the percentage of in-focus regions. Figures 3 (c) and (d) present percentage measures for the stacks of Figure 3(b). Both curves show a smooth trend to increase towards the depth of $1 \mu \mathrm{m}$, indicating the true location of the focal plane, which was verified by a pathologist. Although the classification was done for each image independently, the trend follows closely the expected focus trend for image stacks.

\section{Conclusions}

We proposed an approach to evaluate the focus quality of whole slide images and introduced a way of generating training data from image stacks to train the classifiers. An extensive set of features were extracted and a boosting classifier was trained for classifying images as "in-focus" or "out-of-focus". Our experimental results not only showed that the classifiers trained with the image stacks 
performed better than the limited manually labeled data, but also demonstrated the impressive accuracy of the results and their generalization ability.

\section{References}

1. Liron, Y., Paran, Y., Zatorsky, N.G., Geiger, B., Kam, Z.: Laser autofocusing system for high-resolution cell biological imaging. J. Microsc. 221, 145-151 (2006)

2. Santos, A., Ortiz de Solorzano, C., Vaquero, J.J., Pena, J.M., Malpica, N., del Pozo, F.: Evaluation of autofocus functions in molecular cytogenetic analysis. J. Microsc. 188, 264-272 (1997)

3. Firestone, L., Cook, K., Culp, K., Talsania, N., Preston, K.: Comparison of autofocus methods for automated microscopy. Cytometry 12, 195-206 (1991)

4. Liu, X.Y., Wang, W.H., Sun, Y.: Dynamic evaluation of autofocusing for automated microscopic analysis of blood smear and pap smear. J. Microsc 227, 15-23 (2007)

5. Yeo, T., Jayasooriah, S.H.O., Sinniah, R.: Autofocusing for tissue microscopy. Img. and Vis. Comp. 11, 629-639 (1993)

6. Nayar, S., Nakagawa, Y.: Shape from focus. IEEE PAMI 16(8), 824-831 (1994)

7. Dubois, F., Schockaert, C., Callens, N., Yourassowsky, C.: Focus plane detection criteria in digital holography microscopy by amplitude analysis. Opt. Express 14(13), 5895-5908 (2006)

8. Della Mea, V., Viel, F., Beltrami, C.A.: A pixel-based autofocusing technique for digital histologic and cytologic slides. Comput. Med. Imaging Graph 29, 333-341 (2005)

9. Yazdanfar, S., Kenny, K., Tasimi, K., Corwin, A., Dixon, E., Filkins, R.: Simple and robust image-based autofocusing for digital microscopy. Opt. Express 16, 8670 8677 (2008)

10. Sun, Y., Duthaler, S., Nelson, B.J.: Autofocusing in computer microscopy: selecting the optimal focus algorithm. Microsc. Res. Tech. 65, 139-149 (2004)

11. Padfield, D., Rittscher, J., Roysam, B.: Defocus and low CNR detection for cell tracking applications. In: MIAAB Workshop (2008)

12. Viola, P., Jones, M.: Robust real-time object detection. In: 2nd Int. Workshop on Statistical and Computational Theories of Vision Modeling, Learning, Computing and Sampling (July 2001)

13. Freund, Y., Schapire, R.E.: A decision-theoretic generalization of on-line learning and an application to boosting. Journal of Computer and System Sciences 55(1), 119-139 (1997)

14. Duda, R., Hart, P., Stork, D.: Pattern Classification. John Wiley \& Sons, Chichester (2001)

15. Krotkov, E.: Focusing. IJCV 1(3), 223-237 (1988)

16. Subbarao, M., Choi, T., Nikzad, A.: Focus techniques. J. Opt. Eng. 32, 2824-2836 (1993)

17. Vollath, D.: Automatic focusing by correlative methods. J. Microsc 147, 279-288 (1987)

18. Huang, J., Mumford, D.: Statistics of Natural Images and Models. In: Proceedings IEEE Conference on Computer Vision and Pattern Recognition, pp. 541-547. IEEE Computer Society, Los Alamitos (1999) 